\title{
Determining the Time Needed for the Vortex Method for Preparing Solvent-Free MALDI Samples of Low Molecular Mass Polymers
}

\author{
Scott D. Hanton and James R. Stets \\ Air Products and Chemicals, Inc., Allentown, Pennsylvania, USA
}

Matrix-assisted laser desorption/ionization (MALDI) mass spectrometry is an important technique to characterize many different materials, including synthetic polymers. MALDI mass spectral data is used to determine the polymer average molecular weights, repeat units, and end groups. The development of the vortex method of solvent-free sample preparation showed that remarkably short mixing times could prepare samples that yielded high quality MALDI mass spectra. In this paper, we use microscopy images and MALDI mass spectra to evaluate the mixing time required by the vortex method to produce mass spectra for low molecular mass polymer samples. Our results show that mixing times of as little as $10 \mathrm{~s}$ can generate homogeneous thin films that produce high quality mass spectra with $\mathrm{S} / \mathrm{N} \sim 100$. In addition, ultrashort mixing times of only $2 \mathrm{~s}$ still produce samples with mostly smooth morphology and mass spectra with S/N 10. (J Am Soc Mass Spectrom 2009, 20, 1115-1118) (C) 2009 Published by Elsevier Inc. on behalf of American Society for Mass Spectrometry

$\mathrm{M}$ atrix-Assisted Laser Desorption/Ionization (MALDI) mass spectrometry [1-4] is an important technique to characterize the chemical structure of many different analytes, including industrial polymer materials [5-10]. MALDI generates important data on telomer repeat units, end groups, and average molecular weights of these materials. MALDI methods have been developed to address a broad variety of different polymer materials containing different chemistries and different molecular weight ranges. To address issues with solubility and matrix compatibility, solvent-free sample preparation methods have been developed. While several groups investigated solvent-free sample preparation methods at nearly the same time, the methods developed by Trimpin, Räder, and coworkers have gained widespread use [11-14]. To make the sample preparation step easier, less time consuming, and reduce the risk of cross contamination, we developed a simple version of the solvent-free sample preparation method, now called the vortex method [15]. Previously, we examined the morphology of solvent-free prepared samples using powerful microscopy tools, such as atomic force microscopy (AFM), scanning electron microscopy (SEM), and time-of-flight secondary ion mass spectrometry (TOF-SIMS) [16].

To further understand the vortex method of solventfree MALDI sample preparation, we have studied samples prepared with different vortex mixing times. The

Address reprint requests to Dr. Scott D. Hanton, Air Products and Chemicals, Inc., 7201 Hamilton Blvd., Allentown, PA 18195, USA. E-mail: HantonSD@ airproducts.com previous work showed that $60 \mathrm{~s}$ vortex mixing times produced remarkably homogenous thin films, and that the thin films were composed of submicron features [16]. In this paper, we search for the minimum vortex time required to create these homogenous thin films. We expect that this research will further illuminate the vortex method and enable greater efficiency of preparing these samples by showing that shorter vortexing times create equivalent MALDI mass spectra.

We will characterize the different samples with both MALDI and SEM. MALDI will be the critical method to determine if the sample preparation has been successful, and SEM will be used to learn more about the sample morphology and what changes in the morphology can tell us about the MALDI process.

\section{Experimental}

\section{Chemicals}

The polymethylmethacrylate (PMMA) 2000 Da material was obtained from American Polymer Standards (Mentor, $\mathrm{OH})$. The samples were prepared for MALDI using indole acrylic acid (IAA) obtained from Aldrich (Milwaukee, WI). Samples were doped with sodium trifluoroacetate (NaTFA) as a cationization agent obtained from Aldrich. All chemicals were used as received.

\section{Sample Preparation: Vortex Method}

All samples were prepared using the vortex method as described in references $[16,15]$. In the vortex method,
(C) 2009 Published by Elsevier Inc. on behalf of American Society for Mass Spectrometry. $1044-0305 / 09 / \$ 32.00$

doi:10.1016/j.jasms.2009.02.007
Published online February 12, 2009 Received December 12, 2008 Revised February 3, 2009 Accepted February 4, 2009 
we combined dry components of the analyte (about $0.1-0.2 \mathrm{mg}$ ), matrix (20-40 mg), and cationization agent (trace to $0.1 \mathrm{mg}$ ) in a small glass vial, added two small metal balls, and mixed on a vortex mixer for a variable amount of time. We obtained data on samples mixed for 2-60 s. After mixing, the samples are applied to the MALDI target with a spatula to form a thin film.

\section{Mass Spectrometry}

All of the MALDI experiments were conducted on a Bruker Biflex III (Billerica, MA) TOF mass spectrometer. These experiments were all conducted in reflectron mode using delayed extraction. The Biflex was equipped with a nitrogen laser operated at $337 \mathrm{~nm}$ and $3 \mathrm{~Hz}$. The laser fluence was optimized to be slightly above threshold for ions. The source conditions of the mass spectrometer were optimized for mass resolution near the peak of the analyte telomer distribution. Spectra were collected by moving the sample target relative to the laser and averaging 125 shots/spectrum. The data were analyzed using Polymerix software (Sierra Analytics, Modesto, CA).

\section{Imaging}

The SEM experiments were conducted on a JEOL JSM5910LV scanning electron microscope [17]. The instrument was operated in low-vacuum mode, [low-vacuum mode (sometimes called variable-pressure mode) is used to image insulating specimens without applying a metal coating. The residual air and water molecules in the SEM specimen chamber are ionized by the electron beam, resulting in a positively-charged field immediately above the specimen. The positive field effectively cancels the excess negative charge in the specimen, allowing microscopy to be performed without charging artifacts] with a pressure in the specimen chamber of $\sim 20$ Pascals. No conductive metal coating was applied to the specimens. The accelerating voltage was $20 \mathrm{kV}$ in low-vacuum mode. All images were collected using the backscattered electron signal.
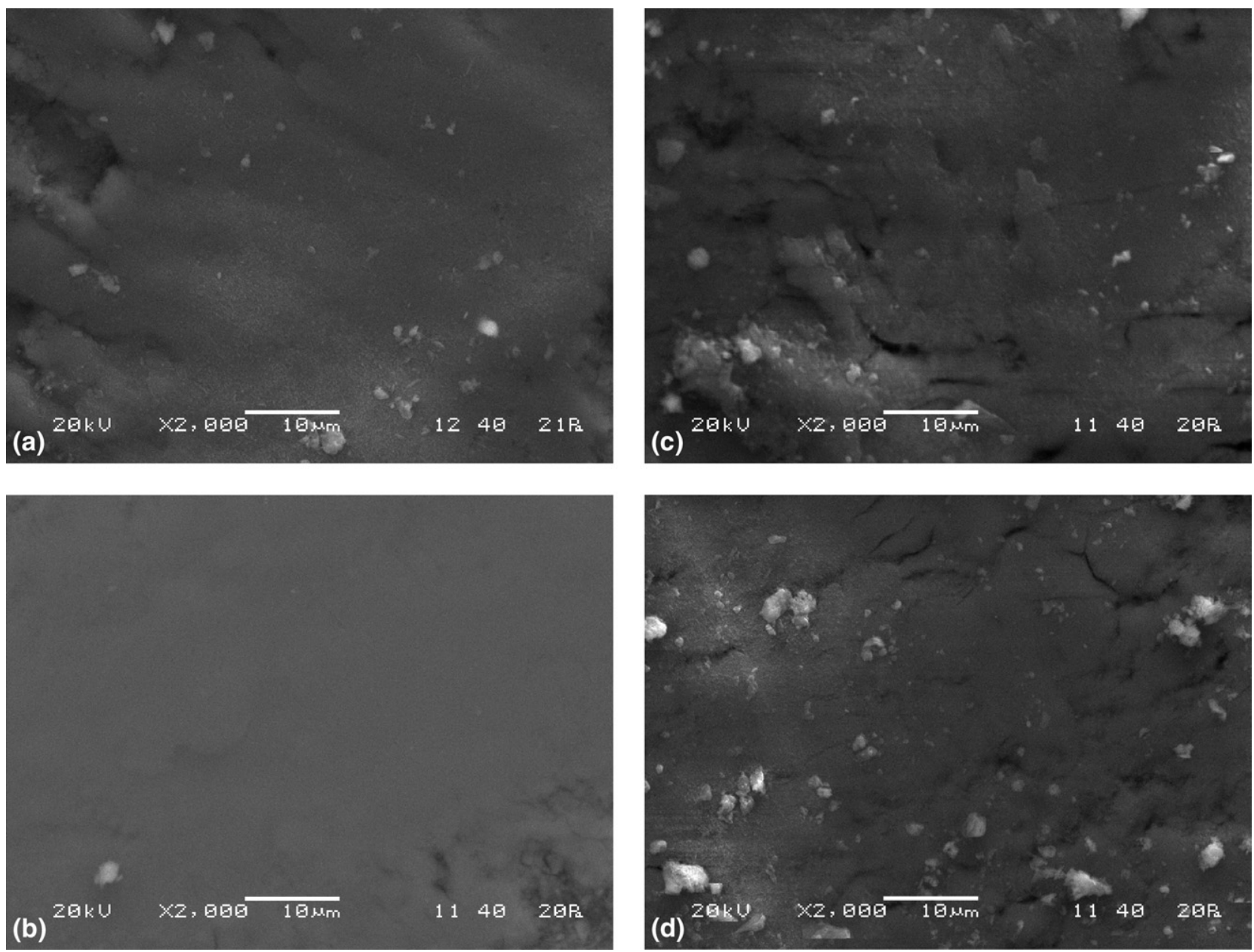

Figure 1. SEM images from samples of PMMA 2000 Da prepared with IAA as the matrix and NaTFA as the cationization agent utilizing 60 (a), 10 (b), 5 (c), and 2 (d) seconds of vortex mixing; 60 and $10 \mathrm{~s}$ samples show very smooth images; 5 and $2 \mathrm{~s}$ samples show more features on the surface. All of the images were acquired at low vacuum with $20 \mathrm{kV}$ accelerating voltage and $2000 \times$ magnification. 


\section{Results and Discussion}

Previous work on vortex method solvent-free sample preparation showed smooth thin films that were surprisingly homogeneous for mixing times of about $60 \mathrm{~s}$ [16]. High and low magnification SEM images of a typical sample are shown in the Supplemental Information, which can be found in the electronic version of this article.

To examine the impact of mixing time on the morphology of the samples, we initially created samples mixed at 60, 50, 40, and $30 \mathrm{~s}$. The SEM images from all of these samples were essentially the same (data not shown) as the images in the Supplemental Information. We then created samples with mixing times of 20, 10, 5, and $2 \mathrm{~s}$. Figure 1 shows the SEM images of the 60, 10, 5, and $2 \mathrm{~s}$ samples. The $10 \mathrm{~s}$ sample (Figure $1 \mathrm{~b}$ ) shows the same featureless thin film as the $60 \mathrm{~s}$ image (Figure 1a). When the mixing time is reduced to $5 \mathrm{~s}$ (Figure 1c) we begin to see more, larger features on the surface of the thin film. The thin film is still complete and has areas that are featureless and homogenous, like the 60 and $10 \mathrm{~s}$ mixing time samples, however, we can now see some other, larger features on the surface. The image of the $2 \mathrm{~s}$ mixing sample (Figure $1 \mathrm{~d}$ ) appears much like the 5 s mixing sample image. We can see morphology differences between the $5 \mathrm{~s}$ and $10 \mathrm{~s}$ of vortex mixing. The samples are rougher and show more and larger features in the 2 and $5 \mathrm{~s}$ samples. From these data we can see that the vortex mixing is starting to be less efficient at $5 \mathrm{~s}$.

We were unable to effectively measure vortex mixing times shorter than $2 \mathrm{~s}$. The issue with any shorter mixing times is having sufficient precision in the timing to demonstrate that the mixing time was clearly different than the $2 \mathrm{~s}$ experiment. If we can determine a method to have precise mixing times less than $2 \mathrm{~s}$, we will continue to explore even shorter mixing times.

While we can start to observe changes, such as greater roughness and larger feature size in the overall morphology at the $5 \mathrm{~s}$ mixing time, how does this morphology translate to the MALDI experiment? We have demonstrated previously that smooth, homogeneous samples produce very effective MALDI mass spectra [16, 18]. We analyzed each of the samples prepared at different mixing times by MALDI. In short, usable data were obtained for each sample, even the $2 \mathrm{~s}$ mixing time sample! Figure 2 shows MALDI mass spectra for the $60,10,5$, and $2 \mathrm{~s}$ mixing time samples. Samples made with mixing times between 60 and $20 \mathrm{~s}$ create very similar MALDI data (data not shown).

Analyzing the mass spectra obtained in these experiments show some interesting results. The mass spectra produced with mixing times from 60-20 s (Figure 2a), and some of the spectra produced with mixing times of $10 \mathrm{~s}$ (Figure 2b) produce high quality data. We measure signal to noise $(\mathrm{S} / \mathrm{N})$ of about 100 . In acquiring the data, (a)

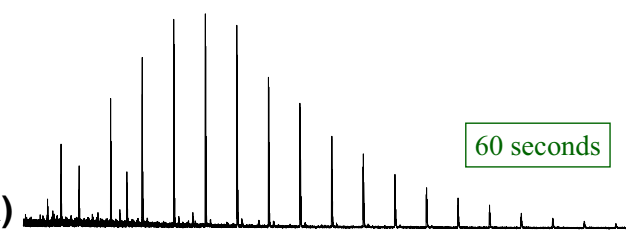

(b)

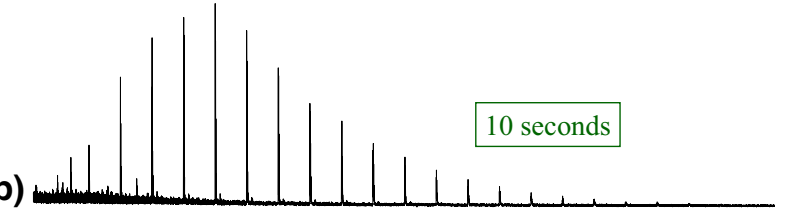

(c)

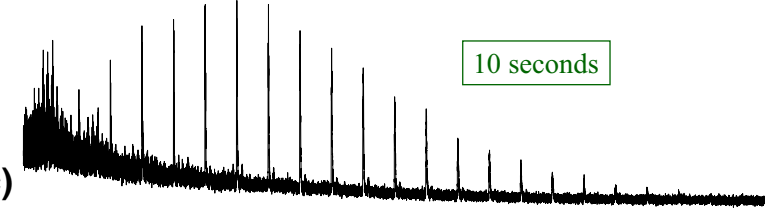

(d)

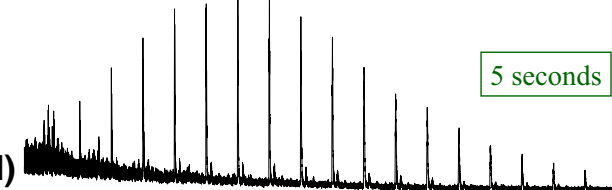

(e)



Figure 2. MALDI mass spectra of samples of PMMA $2000 \mathrm{Da}$ prepared with IAA as the matrix and NaTFA as the cationization agent utilizing 60 (a), 10 (b) and (c), 5 (d), and 2 (e) seconds of vortex mixing. We see excellent mass spectra with $\mathrm{S} / \mathrm{N} \sim 100$ for spectra from $60-10 \mathrm{~s}$, good mass spectra with $S / N \sim 50$ for spectra from 10-5 s, and meaningful mass spectra with $S / N \sim 10$ for spectra from the ultrashort mixing time of $2 \mathrm{~s}$.

we find that the samples are very homogeneous in that we see essentially no dependence of sample position on the MALDI signal. This was also observed in the previous work, [16] and in work on samples produced by electrospray deposition [18]. The mass spectra produced with mixing times from 10-5 s (Figure $2 \mathrm{c}$ and d) can still produce very nice MALDI data. We measure $\mathrm{S} / \mathrm{N} \sim 50$, but now we do observe some spot to spot differences, and some sample to sample differences. For example, Figure $2 \mathrm{~b}$ and $\mathrm{c}$ are both from the same sample produced with $10 \mathrm{~s}$ of mixing time. The mass spectrum in Figure $2 b$ is excellent, much more like the mass spectra obtained from longer mixing times. Figure $2 \mathrm{c}$ demonstrates the range of data that can be produced with the shorter mixing times. In fact, Figure $2 b$ is not as 
good a mass spectrum as that from a sample produced from only $5 \mathrm{~s}$ of mixing time (Figure $2 \mathrm{~d}$ ).

Even at a mixing time of only $2 \mathrm{~s}$, we still obtain a usable mass spectrum of the PMMA 2000 Da sample. The $\mathrm{S} / \mathrm{N}$ is now only about 10 and we now observe some significant spot to spot variations. While Figure 2e is not an outstanding mass spectrum, it clearly shows that the mixing process in the vortex method is remarkably fast. In only $2 \mathrm{~s}$ of mixing time, we have created sufficient interaction between the matrix and the analyte to produce a usable (if not high quality) MALDI mass spectrum.

\section{Conclusions}

Using MALDI and SEM, we explored the time dependence of the vortex mixing of the solvent-free MALDI sample preparation method. Our data shows that the vortex method of sample preparation is effective with surprisingly short mixing times. We obtain high quality data from samples with as little as $10 \mathrm{~s}$ of mixing time. For even shorter mixing times of 5 , or even $2 \mathrm{~s}$, we can obtain meaningful mass spectra of the polymer analyte.

In this work, we have focused on low mass polymers in the mass range below $5000 \mathrm{Da}$. Others have shown that higher mass polymers can be successfully prepared by solvent-free methods. We are still working to understand the impact of the average molecular weight and the mixing time. At this point, apparently larger polymers will benefit from longer mixing times, and that these ultrashort mixing times are not applicable to higher mass polymers.

\section{Acknowledgments}

The authors thank Air Products and Chemicals, Inc., for their support of this research, Professor Kevin Owens from Drexel University for helpful discussions about these experiments, Dr. Sarah Trimpin from Wayne State University for helpful discussions of her solvent-free sample preparation technique, and Dr. Sherri Bassner for critical review of the manuscript.

\section{Appendix A Supplementary Material}

Supplementary material associated with this article may be found in the online version at doi:10.1016/ j.jasms.2009.02.007.

\section{References}

1. Tanaka, K.; Waki, H.; Ido, Y.; Akita, S.; Yoshido, Y.; Yoshido, T. Protein and Polymer Analyses up to $\mathrm{m} / \mathrm{z} 100,000$ by Laser Ionization Time-of-flight Mass Spectrometry. Rapid Commun. Mass Spectrom. 1988, 2, 151-153.

2. Karas, M.; Hillenkamp, F. Laser Desorption Ionization of Proteins with Molecular Masses Exceeding 10,000 Daltons. Anal. Chem. 1988, 60, 2299 2301.

3. Bahr, U.; Deppe, A.; Karas, M.; Hillenkamp, F.; Giessman U. Mass Spectrometry of Synthetic Polymers by UV-Matrix-Assisted Laser Desorption/Ionization. Anal. Chem. 1992, 64, 2866-2869.

4. Danis, P.; Karr, D.; Mayer, F.; Holle, A.; Watson, C. The Analysis of Water-Soluble Polymers by Matrix-Assisted Laser Desorption Time-ofFlight Mass Spectrometry. Org. Mass Spectrom. 1992, 27, 843-846.

5. Hanton, S. D. Mass Spectrometry of Polymers and Polymer Surfaces. Chem. Rev. 2001, 101(2), 527-569.

6. Nielen, M. W. F. MALDI Time-of-Flight Mass Spectrometry of Synthetic Polymers. Mass Spectrom. Rev. 1999, 18, 309-344.

7. Montaudo, G.; Lattimer, R. P., Eds; Mass Spectrometry of Polymers; CRC Press: Boca Raton, FL, 2002.

8. Pash, H.; Schrepp, W., Ed; MALDI-TOF Mass Spectrometry of Synthetic Polymers; Springer-Verlag: Berlin, 2003.

9. Wallace, W. E.; Guttman, C. M.; Hanton, S. D. Quantitative Synthetic Polymer Mass Spectrometry Workshop. J. Res. Natl. Inst. Stand. Technol. 2003, 108, 79-85.

10. Wallace, W. E.; Guttman, C. M.; Wetzel, S. J.; Hanton, S. D. Mass Spectrometry of Synthetic-Polymer Mixtures Workshop. Rapid Commun. Mass Spectrom. 2004, 18, 518-521.

11. Przybilla, L.; Brand, J. -D.; Yoshimura, K.; Räder, H. J.; Müllen, K. MALDI-TOF Mass Spectrometry of Insoluble Giant Polycyclic Aromatic Hydrocarbons by a New Method of Sample Preparation. Anal. Chem. 2000, 72, 4591-4597.

12. Trimpin, S.; Rouhanipour, A.; Räder, H. J.; Müllen, K. New Aspects in Matrix-Assisted Laser Desorption/Ionization Time-of-Flight Mass Spectrometry: A Universal Solvent-Free Sample Preparation. Rapid Commun. Mass Spectrom. 2001, 15, 1364-1373.

13. Trimpin, S.; Keune, S.; Räder, H. J.; Müllen, K. Solvent-Free MALDI-MS Developmental Improvements in the Reliability and the Potential of MALDI in the Analysis of Synthetic Polymers and Giant Organic Molecules. J. Am. Soc. Mass Spectrom. 2006, 17, 661-671.

14. Trimpin, S.; Deinzer, M. L. Solvent-free MALDI-MS for the Analysis of a Membrane Protein Via the Mini Ball Mill Approach: Case Study of Bacteriorhodopsin. Anal. Chem. 2007, 79, 71-78.

15. Hanton, S. D.; Parees, D. M. Extending the Solvent-Free MALDI Sample Preparation Method. J. Am. Soc. Mass Spectrom. 2005, 16, 90-93.

16. Hanton, S. D.; McEvoy, T. M.; Stets, J. R. Imaging the Morphology of Solvent-Free Prepared MALDI Samples. J. Am. Soc. Mass Spectrom. 2008, 19, 874-881.

17. Newbury, D. E.; Joy, D. C.; Echlin, P.; Fiori, C. E.; Goldstein, J. I. Advanced Scanning Electron Microscopy and X-Ray Microanalysis; Plenum Press: New York, 1986.

18. Hanton, S. D.; Hyder, I. Z.; Stets, J. R.; Owens, K. G.; Blair, W. R. Guttman, C. M.; Giuseppetti, A. A. Investigations of Electrospray Sample Deposition for Polymer MALDI Mass Spectrometry. J. Am. Soc. Mass Spectrom. 2004, 15, 168-179. 